ÉGYPTE monde arabe

\section{Égypte/Monde arabe}

4 | 1990

Démocratie et démocratisation dans le monde arabe

\title{
Les islamistes et la transition démocratique. Jalons pour une recherche (extraits)
}

\section{François Burgat}

\section{(2) OpenEdition}

\section{Journals}

Édition électronique

URL : https://journals.openedition.org/ema/351

DOI : 10.4000/ema.351

ISSN : 2090-7273

Éditeur

CEDEJ - Centre d'études et de documentation économiques juridiques et sociales

Édition imprimée

Date de publication : 31 décembre 1990

ISSN : 1110-5097

Référence électronique

François Burgat, "Les islamistes et la transition démocratique. Jalons pour une recherche (extraits) », Égypte/Monde arabe [En ligne], 4 | 1990, mis en ligne le 08 juillet 2008, consulté le 07 juillet 2022. URL: http://journals.openedition.org/ema/351 ; DOl : https://doi.org/10.4000/ema.351

Ce document a été généré automatiquement le 7 juillet 2022.

Tous droits réservés 


\title{
Les islamistes et la transition démocratique. Jalons pour une recherche (extraits)
}

\author{
François Burgat
}

1 Dans le monde arabe, la relève en cours des régimes issus des mouvements nationaux implique l'articulation, aux systèmes institutionnels existants, de forces politiques dont la gestation s'est, pour une part au moins, amorcée en dehors de ces systèmes et, le cas échéant, loin de (et parfois contre) les énoncés théoriques qui fondent aujourd'hui leur fonctionnement.

2 Les courants islamistes, dont la montée en puissance s'est opérée un peu partout dans le monde arabe au cours de la décennie écoulée, constituent l'archétype de ces nouveaux acteurs dont la présence est très souvent perçue comme antinomique avec le processus de (re)distribution démocratique du pouvoir.

3 Pour bon nombre d'observateurs, aussi bien arabes qu'occidentaux, l'adhésion proclamée des leaders de ces courants aux valeurs pluralistes au nom desquelles s'opère cette transition a tout lieu d'être mise en doute. Pour les gouvernants, cette hypothèse cautionne implicitement la lenteur du processus de démocratisation et, le cas échéant, sa sélectivité. En Égypte, au Maroc et en Tunisie notamment, les formations islamistes sont ainsi tenues à l'écart de la scène politique légale.

4 Pour l'essentiel, le raisonnement qui fonde cette réticence s'appuie sur une triple assertion :

- l'absence, dans la pensée musulmane classique et notamment dans la notion de chûra (consultation), de référence explicite aux formes institutionnelles qui fondent la notion moderne d'opposition politique,

- la lenteur avec laquelle les leaders islamistes se sont démarqués du refus du principe démocratique (longtemps considéré comme incompatible avec le monopole des oulémas de l'islam et de nature à diviser la communauté des croyants) exprimé explicitement par leurs premiers théoriciens, Abu 'Ala al-Mawdûdî et Hasan al-Banna notamment ${ }^{1}$, 
- les pratiques antidémocratiques, individuelles ou collectives, oppositionnelles ou (en Iran) gouvernementales, qui seraient l'apanage du comportement des militants islamistes modernes.

5 Cette première hypothèse ne saurait aucunement être écartée du champ des possibles. Mais elle ne saurait non plus être, sans danger, érigée en une grille d'analyse unique qui conduirait à ne mesurer les chances de la démocratie arabe qu'à l'aune du talent des régimes en place à combattre leurs islamistes. Hypothèse unique, elle tend en fait à perdre son statut pour acquérir celui d'un postulat, équivalent dès lors, vis-à-vis de la démarche cognitive, à ce que le parti unique est à la démocratie.

6 Au-delà de ce premier vice de forme, l'hypothèse de l'absolue incompatibilité des Forces islamistes avec l'univers des référents de la pensée politique libérale n'est pas non plus à l'abri de critiques de fond.

7 Parce que l'institutionnalisation de la représentation pluraliste des courants politiques est un produit récent de la pensée libérale occidentale, la tentation est grande d'en extrapoler l'idée que la pensée musulmane classique est forcément demeurée intrinsèquement étrangère à la notion d'une opposition politique légitime dans sa tâche de limitation du pouvoir et que la "doctrine de l'islam » (dont les islamistes seraient censés ici être les héritiers naturels et les gardiens vigilants) les empêcherait d'intérioriser dès lors les catégories indispensables à la construction de l'univers institutionnel de la démocratie.

8 Cette appréciation doit tout d'abord être relativisée. En droit, même si la régulation du pouvoir du Prince par une instance pluraliste institutionnalisée paraît bien être demeurée étrangère au droit musulman classique, jamais le pouvoir du Prince musulman, d'ordre contractuel puisque fondé sur le contrat de ba'ya passé avec la communauté, n'a été pour autant considéré comme illimité. L'idée d'une possible sanction, le plus souvent la révocation et la mort, étaient bien présentes dans l'univers « institutionnel» islamique. Elle s'exprimait toutefois à l'intérieur de la culture politique dominante, qui était alors une culture religieuse, et prenait donc comme critère de la légalité de l'action du Prince non point tant l'avis d'une majorité de ses sujets que la capacité de ce Prince à ne point enfreindre une norme dite "divine ». L'interprétation de cette légalité « divine » n'en était pas moins confiée pourtant à des intermédiaires parfaitement humains, à qui la théorie politique reconnaissait le droit, s'ils parvenaient à gagner à leur point de vue la majorité de la «casse politique » de l'époque, de décider de mettre un terme aux débordements du pouvoir. Mais le cœur du débat n'est sans doute pas là.

\section{Islamisme et démocratie : la dynamique de la réappropriation}

9 Assurément, plusieurs siècles plus tard, les premières constructions doctrinales islamistes se sont érigées sur le refus plus ou moins explicite du principe démocratique. Parce qu'il remettait en cause l'omnipotence des oulémas-guides de la communauté. Parce qu'il impliquait la constitution de cette communauté en partis et donc, au mépris du sacro-saint principe d'unité, sa division. Parce que l'idée d'une législation humaine contredisait la lecture littéraliste du principe de la primauté de la norme divine et celui de la vocation du Coran et de la Sunna à satisfaire la totalité des besoins normatifs de la 
communauté. Plus conjoncturellement, parce que le vocabulaire démocratique a longtemps été identifié à l'héritage et aux régimes de cet Occident (puis à ses " relais " supposés à l'intérieur du monde arabe) dont la thématique islamiste avait précisément pour logique de fonctionnement de se démarquer. Et .enfin parce que l'image qui se dégage de l'usage très sélectif de ces référents que pratiquent actuellement les régimes arabes n'a pas non plus contribué à leur valorisation.

C'est notamment en ces termes que s'explicite parfois encore aujourd'hui la défiance de certains leaders islamistes à l'égard du concept de démocratie. Ainsi de l'Algérien Ali Belhaj, considéré comme le plus radical des porte-paroles du Front Islamique de Salut. Il avait salué la performance de son parti aux élections locales du 12 juin 1990 en précisant qu'elle n'était "pas une victoire de la démocratie mais une victoire de l'islam » et il refuse généralement de faire référence au concept de démocratie :

La démocratie est un slogan repris par les "démocrates' », les démocrates entre guillemets. C'est un concept assez flou que beaucoup utilisent pour se grandir. (...) La question est de savoir quel est le modèle politique qui peut constituer une alternative au régime actuel. Sinon, pourquoi nous opposer à ce régime? Or ce régime se dit démocratique, il s'appelle République algérienne démocratique et populaire. Cela nous amène à dire que le slogan 'démocratie' prôné par certains n'est qu'une clownerie (...). Notre compréhension de la démocratie, c'est de permettre au peuple de donner son point de vue. Notre pays vit aujourd'hui une situation de crise politique. Le dialogué est donc pour nous le meilleur moyen d'aboutir. Si la démocratie signifie débat et respect, alors nous sommes pour. ${ }^{2}$.

11 A l'extrême opposé du registre islamiste maghrébin, l'influent leader du Parti tunisien de la Renaissance (Hizb al-Nahda), Rached Ghannouchi, souligne lui aussi cette contradiction du discours des régimes à l'égard de leurs opposants islamistes, mais il est explicitement acquis pour sa part au vocabulaire de la démocratie ${ }^{3}$. Son propos explicite à peu de chose près l'argumentaire moyen des islamistes en mal de reconnaissance légale :

L'ennui c'est que, s'agissant de démocratie et de droits de l'homme, les états de service de la plupart des accusateurs laissent passablement sceptique... (Ils) n'ont cessé de persécuter les libertés, de truquer les élections, de monopoliser l'information. On baigne dans l'absurdité... (...). La vérité est simple. Nos adversaires ont toujours eu du mal à s'accommoder de la démocratie et du pluripartisme. Longtemps, ils les ont refusés ouvertement, de façon explicite et aujourd'hui ils le font honteusement, de façon déguisée. Ils n'acceptent le jeu démocratique qu'à condition qu'ils en soient, en toute hypothèse, les gagnants. Je vais plus loin: supposons que nous soyons des antidémocrates invétérés et nos adversaires des démocrates irréprochables. Ils pourraient, s'ils étaient sincères, nous contraindre à jouer le jeu de la démocratie. En France, les sentiments démocratiques de l'extrême droite (comme d'ailleurs de l'extrême gauche) ne sont pas évidents, mais nul ne songe à l'exclure de la démocratie française. De même pour la démocratie israélienne ou pour les démocraties américaine, britannique, allemande, etc. C'est grâce au jeu démocratique que tous les partis politiques nourris d'idéologies autoritaires ont été, dans tous les pays, intégrés sinon marginalisés. Donc. ne seraitce que pour nous rééduquer, on devrait nous faire une place dans la "démocratie". Il y a (en Tunisie) un parti communiste. On ne lui a pas demandé de renier le marxisme, idéologie démocratique s'il en est, pour être reconnu. On brandit en fait cette exigence uniquement contre nous parce que l'on cherche à se débarrasser d'un parti fort et puissant. ${ }^{4}$

12 De fait, l'adhésion de la plupart des leaders islamistes aux principes du pluralisme démocratique est désormais explicite. Chez certains d'entre eux, Rached Ghannouchi 
tout particulièrement mais pas seulement ${ }^{5}$, elle est argumentée autrement qu'en termes de simple tactique et avec une constance qui ne peut pas être perpétuellement méconnue, sauf à vouloir tenter d'accréditer l'idée d'une antinomie générale, structurelle et irréversible entre les forces en gestation dans la mouvance islamiste et l'émergence de systèmes démocratiques. Or cette position est précisément tout aussi « idéologique » et arbitraire que celle qui consiste à donner crédit, en sens inverse, à la surenchère démocratique du discours d'une partie de ces forces.

Bien davantage que l'évaluation des pratiques juridiques des sociétés musulmanes de l'âge classique ou même, plus près de nous, celles des fondateurs de la nouvelle thématique islamiste, c'est bien la réaffirmation de la capacité de la communauté musulmane, composante "islamiste » incluse, à faire évoluer son système normatif tout en le maintenant en référence avec un dogme considéré comme intangible, qu'il est en effet nécessaire de réintroduire dans le débat.

En faisant de ceux qui se réclament de l'islam une espèce vouée à être éternellement déconnectée de l'univers des valeurs démocratiques, le regard extérieur manifeste un refus d'historiciser dont le paradoxe veut qu'il soit très comparable à celui qui est légitimement reproché à la démarche islamiste: en intériorisant la supposée intangibilité des comportements politiques liés à la religion musulmane, il cautionne la pseudo-intemporalité des expressions normatives de l'islam qui est le fondement même de la thématique islamiste. C'est ce même refus de l'histoire qui nourrit en effet la demande incantatoire de retour à l'antique chari'a qui tient lieu de programme aux moins éclairés des émules de l'Imam de Qom. En déniant aux islamistes toute capacité à évoluer, c'est à la vision hautement idéologique que ces islamistes ont d'eux-mêmes et de leur système normatif que se soumet le regard de ceux qui entendent les critiquer.

Quatorze siècles d'histoire musulmane ont pourtant amplement démontré que, sous le pavillon de l'islam, n'ont jamais navigué (hormis dans le champ limité des pratiques "cultuelles») que des adaptations circonstancielles du dogme aux exigences sociales d'un lieu et d'un siècle donnés ${ }^{6}$. Sans doute le croisement de la religion musulmane et de la mobilisation politique s'est-il partais exprimé sur le registre de la violence. Sans doute y a-t-il eu (par exemple à Téhéran) et très vraisemblablement y aura-t-il encore des régimes pour tenter, tout comme leurs prédécesseurs l'ont fait avec les valeurs nationalistes, de donner à leur autoritarisme la caution de l'Islam. Mais il n'en serait pas moins hasardeux de faire du recours au vocabulaire de la religion le corollaire de l'émergence obligée des nouveaux totalitarismes. Tout comme il serait extrêmement difficile de démontrer, même si le regard académique tarde parfois à empêcher le sens commun de le croire, que la violence politique a été en terre arabe le corollaire de l'apparition des seuls courants " religieux ", alors qu'elle a sans doute été au contraire (entre chrétiens ou musulmans, opposants ou gouvernants, laïcs ou religieux, de gauche ou de droite etc...) la seule denrée politique équitablement répartie. Sans doute la prétention totalisante des religions monothéistes recèle-t-elle un potentiel totalitaire qui est en islam d'autant plus fort que la barrière de la sécularisation y est, au moins en principe, réfutée. Mais de l'Inquisition catholique aux goulags staliniens, l'histoire mondiale est là pour attester que, bien davantage que leur contenu intrinsèque, ce sont les conditions d'appropriation sociale des dogmes en tous genres qui ont toujours déterminé le niveau de violence politique dont ils pouvaient, le cas échéant, « couvrir » le déploiement. 

de souligner la relativité des catégories forgées par l'Occident pour hiérarchiser ses solidarités politiques en terre arabe ou musulmane. La violence de l'agression perpétrée au Koweït par I'« allié laïc irakien » des années 80 prouve à tout le moins que le comportement des acteurs politiques n'est pas seulement inhérent à la nature de leurs référents idéologiques, et que la propension d'un régime à s'abstenir de recourir, à l'intérieur où à l'extérieur de ses frontières, à la violence politique, n'est pas mesurable à sa seule réticence à l'égard de la terminologie islamiste. L'erreur consisterait dès lors à ériger en butoirs intemporels des expressions dont l'histoire récente des courants islamistes permet déjà d'entrevoir qu'elles ne sont que les jalons d'un processus de gestation. islamiste, supposée souffrir des mêmes limites que la pensée musulmane classique), la perception figée d'une réalité qui ne l'est pas aboutit en fait à, focaliser le regard sur le vocabulaire de l'appropriation du politique - que la thématique islamiste a précisément pour fonction de renouveler - au détriment des conditions sociales, variables dans le temps et dans l'espace, de cette appropriation. Bien davantage que son vocabulaire, ce sont les conditions de son appropriation sociale qui sont susceptibles en effet d'affecter les modes d'expression de la thématique islamiste. Ce sont donc ces paramètres sociohistoriques qui, bien plus vraisemblablement que la thématique islamiste elle-même, recèlent aujourd'hui l'inconnue de la transition démocratique arabe. politique arabe peut être attestée, la détermination des conditions à venir d'exploitation de ce capital et notamment la nature plus ou moins autoritaire ou démocratique des régimes qu'ils sont susceptibles de mettre en œuvre demeure en effet aujourd'hui très largement aléatoire. Elle échappe en tout état de cause au seul critère $\mathrm{du}$ recours au vocabulaire de la religion, pour relever dès lors de paramètres plus nombreux et d'un maniement plus complexe qui permettent de caractériser les formes $\mathrm{du}$ développement politique qui a produit ces forces et l'environnement socioéconomique mais aussi politique dans lequel elles évoluent: au-delà de la seule quantification incantatoire de la «menace islamiste » qui borne trop souvent le champ des interrogations, c'est sur l'environnement socio-historique des forces islamistes, très différencié d'un pays à l'autre, que doit plus efficacement se mobiliser l'analyse.

Le fait que le discours islamiste se serve de l'héritage culturel occidental comme d'un repoussoir et fonctionne de manière privilégiée avec des catégories exprimant méfiance et défiance à l'égard de l'Occident ne doit pas masquer en effet que sa logique profonde est sans doute plus proche d'un processus de réappropriation, fut-elle sélective, que d'un rejet. Alors que les conditions de leur irruption dans la foulée de l'expansion coloniale avaient, au XIXe siècle, rendu les référents "occidentaux" inappropriables par des sociétés dont les codes culturels s'étaient trouvés brutalement marginalisés, la réintroduction islamiste de la culture du père pourrait même très paradoxalement réussir ce que ni la violence coloniale ni la contre-violence nationaliste n'avaient pu mener à bien : la conciliation de codes culturels des sociétés anciennement colonisées avec l'essentiel de ces valeurs que l'irruption occidentale y avait introduites sans parvenir pour autant à assurer, hors de l'élite acculturée, les conditions de leur appropriation et partant celle de leur effectivité. 


\section{Chari'a et laïcité : les frontières de la réappropriation}

Jusqu'où la réappropriation de l'univers laïque de la pensée démocratique occidentale par une pensée d'obédience religieuse peut-elle s'opérer? Si l'on veut considérer que l'essence de la démocratie réside dans le principe du primat de la volonté humaine en matière normative, il existe bel et bien une frontière explicite entre les univers « occidentalo-laïque » et «arabe-religieux ». Encore faut-il, pour en prendre l'exacte mesure, vérifier que la pensée politique occidentale fonde bien l'absolu libre arbitre de l'homme dans la détermination des lois qui le gouvernent. Ou, plus exactement, que la pratique occidentale a effectivement érigé ce principe théorique en norme absolue. Or il semble qu'à de rares exceptions, cette vision doive être nuancée. Des USA ( In God we trust!») à la France républicaine en passant par la Grande-Bretagne, les expériences phares de la pensée libérale occidentale ont toujours admis en effet l'existence de principes supérieurs à la volonté humaine, fut-elle collective. Dans bon nombre de cas, et non des moindres, la référence à la loi divine est même demeurée explicite : c'est le cas des USA et de l'Allemagne fédérale. Presque partout ailleurs, elle ne s'est absentée que formellement, "principes généraux du droit» et autre «droit naturel » attestant que quand bien même serait-elle celle d'une majorité d'entre eux, la volonté des hommes était soumise à un corpus de référents universels qu'elle n'était pas habilitée à remettre en cause.

21 Sans doute l'absence de référence explicite à l'origine divine de ce corpus normatif marque-t-elle bien une différence. Mais cette différence elle-même doit être largement relativisée: elle est le résultat de la forme et du contexte de sa formulation l'émancipation de la tutelle ecclésiastique - tout autant que la volonté d'affirmer un principe essentiel.

Les dangers du «majoritarisme » (c'est-à-dire la tentation de forces politiques dotées d'une solide majorité de gouverner en méconnaissance des intérêts de la minorité) - qui pourrait résulter de l'arrivée au pouvoir de forces peu susceptibles de respecter les exigences démocratiques nouvellement incluses dans leur discours - ne doivent certes pas être sous-estimées. Mais la présence de forces maniant la thématique islamiste ne saurait être considérée comme le critère unique, en l'occurrence négatif, d'évaluation d'un processus de démocratisation où le reste de la classe politique, miraculeusement absous de décennies d'autoritarisme, se verrait par sa seule prétention à se différencier de la mouvance islamiste, habilité à porter le flambeau des libertés individuelles et collectives et reconnaître toute latitude de se préserver, à n'importe quel prix, de ses concurrents.

Parce que .leur présence en est l'une des composantes, la participation des forces islamistes apparaît au contraire comme l'une des conditions sine qua non d'une transition démocratique qui perdrait toute signification en l'absence, et a fortiori " contre " leur entière génération, dont rien ne permet sérieusement de préjuger aujourd'hui le rôle à venir - frein ici mais pourquoi pas partie prenante ailleurs - qu'ils pourront y jouer. 


\section{Algérie, Égypte, Yémen, Jordanie : des itinéraires différenciés}

Bien davantage ${ }^{7}$ que dans l'exégèse de leurs discours et a fortiori de celui de leurs ancêtres réels ou supposés, l'évaluation de la capacité des acteurs que la poussée islamiste rapproché ici ou là de la scène politique légitime à adopter des conduites démocratiques passe avant tout par l'analyse du contexte de la production de ces acteurs, soit l'analyse socio-historique de la formation sociale dont ils sont issus et, tout autant, celle des régimes dont ils contestent le monopole. Algérie, Tunisie, Égypte, Jordanie, Yémen donnent de ce point de vue autant de réponses différenciées. Si les ressources politiques qu'exploitent aujourd'hui les islamistes sont bien "disponibles" partout dans un monde arabe qui a tout entier, au siècle dernier, pris brutalement contact avec le système de représentation de l'Occident, d'une société à l'autre, les conditions historiques de cette rencontre ont en effet varié considérablement et ses conséquences, notamment «identitaires", n'ont pas eu partout la même dimension. Des trois années de l'expédition scientifico-militaire du général Bonaparte en Égypte aux 132 années de colonisation de peuplement de l'Algérie, du protectorat (Tunisie, Maroc, Égypte) au mandat (Syrie, Liban, Jordanie ou Irak, etc.), les formes politicojuridiques et la durée de la présence occidentale (dans des sociétés qui étaient ellesmêmes plus ou moins bien armées pour résister à la concurrence culturelle de l'étranger) ont notablement varié. L'attitude des élites au pouvoir au lendemain des indépendances a considérablement accru, ensuite, ces premières différences: monarchies renouant avec les symboles du régime précolonial, ou Républiques laïques balayant les anciennes structures ottomanes ont, selon les cas, satisfait certains compartiments de la demande identitaire ou au contraire laissé largement ouvert l'espace de sa manifestation sous une forme contestataire. Sans nulle part empêcher l'extension du territoire de fonctionnement des codes culturels occidentaux ainsi qu'un certain processus de laïcisation, les élites postcoloniales n'ont pas puisé partout au même rythme dans l'un ou l'autre réservoir «traditionnel» ou "occidentalomodemiste " des référents politiques. Ainsi de l'arabisme, c'est-à-dire de l'identification aux valeurs linguistiques et culturelles arabes mais aussi de l'idéologie unitaire arabe. Ainsi bien sûr et surtout des valeurs religieuses, centrales dans le dispositif de légitimation des monarchies marocaine ou du Golfe, perçues comme facteur d'immobilisme social ailleurs (Tunisie, Algérie, Syrie, Irak notamment) et tenues comme telles à la périphérie du discours politique. Qu'elle ait été le fait des régimes ou de leurs opposants, l'aspiration diffuse au renforcement de l'identité s'est donc manifestée dans le temps et dans l'espace arabo-musulman à un rythme et avec une intensité qui ont pris en compte ces différences.

La caractéristique essentielle du terroir algérien n'est pas tant, de ce point de vue, d'avoir produit le plus fort potentiel de mobilisation islamiste de la région que - ceci expliquant cela - d'apparaître en la matière comme un archétype. La colonisation de peuplement y a duré plus d'un siècle. L'acculturation (notamment linguistique) y a été plus poussée que partout ailleurs. Postérieurement à l'indépendance, la "désislamisation culturelle » a été particulièrement accentuée.

Comparée aux terroirs maghrébins de la mobilisation islamiste, l'Égypte recèle quant à elle des particularismes qui, à un degré ou à un autre, sont communs aux terroirs du Machreq arabe, à tout le moins à ceux (Liban, Syrie, Jordanie, Irak) du croissant fertile. 
La question identitaire est vraisemblablement moins aiguë en effet au Machreq et tout particulièrement en Égypte, qu'elle ne l'est au Maghreb et tout particulièrement en Algérie. Ce différentiel tient aux ressources historiques propres de ces sociétés. Contrairement à la situation qui prévaut au Maghreb, les sociétés recèlent au Machreq une " histoire longue " susceptible de fournir des référents préislamiques forts ${ }^{8}$. Il tient ensuite aux modalités historiques de leur entrée en contact avec la modernité, modalités qui déterminent pour une part au moins le contenu de l'imaginaire de la relation à l'Occident. Au Machreq, la prétention du référent islamique à l'universalisme est enfin battue en brèche (à moins qu'elle ne soit exacerbée ?) par la présence d'un référent chrétien dont (au regard des catégories de la mobilisation nationaliste) la légitimité ne peut pas (comme c'est le cas pour la très embryonnaire église maghrébine) être mise en doute.

Ce paradigme « oriental » devra donc être comparé à son homologue maghrébin, tout particulièrement à I'« archétype algérien", en même temps que les tendances plus récentes de l'expression institutionnelle du mouvement islamiste strictement égyptien (et notamment la nature du dualisme au moins apparent qui s'y manifeste entre la tendance légaliste des Frères Musulmans et leurs challengers du Groupe Islamique) devront être précisées.

La Jordanie illustre notamment la situation d'un courant islamiste dont la principale composante au moins ne s'est jamais véritablement opposée au régime. Pour combattre les idéologies populistes laicïsantes (baassisme et nassérisme) qui menaçaient l'assise idéologique de son pouvoir, le trône hachémite s'est au contraire appuyé sur la puissante association des Frères Musulmans 9 .

Le Yémen réunifié présente enfin pour sa part l'intérêt considérable d'être le produit de deux terroirs dont les itinéraires de modernisation (un Nord montagneux et tribal qui n'a connu comme de présence étrangère qu'ottomane et égyptienne ${ }^{10}$ et un Sud qui a subi une présence britannique directe et connu comme le seul régime arabe à s'être explicitement réclamé du marxisme léninisme) constituent les deux types opposés.

Les modalités historiques de la relation à l'Occident, les politiques culturelles conduites par les élites au pouvoir au lendemain des indépendances, leur position dans le champ religieux (c'est-à-dire à la fois leur réaction face à la poussée islamiste et l'espace symbolique qu'elles ont laissé libre à son déploiement), le paysage économique également, dont l'évolution, au gré des poussées de frustration sociale, a souvent accéléré la révélation des « carences identitaires ", stimulé la recherche de vecteurs de contestation mais aussi affecté, notamment par le biais des phénomènes migratoires, le rôle des influences extérieures etc... constituent donc autant de paramètres dont la prise en compte doit impérativement venir troubler la trop rapide antinomie de la relation analytique entre I'« islamisme » et la " démocratie ».

31 Cette analyse s'inscrit, dans le cadre du CEDEJ, au sein d'une démarche dont l'objectif est d'analyser les conditions d'émergence, dans le monde arabe, des formations islamistes et le processus de leur articulation à la scène politique légitime. Elle prolonge et élargit (au Yémen réunifié, à la Jordanie et à l'Égypte notamment) une recherche initiée à l'IREMAM d'Aix-en-Provence, dont une première formalisation a été donnée in L'islamisme au Maghreb: la voix du Sud, Karthala, Paris, 1988. Les prémisses du second volet de cette anal/se ont fait l'objet de publications partielles in (notamment) Relazioni internazionali ( $\mathrm{N}^{\circ}$ 3) 1989 (Milan), L'État du Monde 1990 (Paris), Maghreb Machreq-Monde Arabe, septembre 1990 et, s'agissant plus particulièrement de la problématique 
"Islamisme et Démocratie ", d'une communication au colloque "Elections, participation et transition démocratique en Afrique du Nord ", Université autonome de Madrid, 12 mai 1990. La partie consacrée à l'inventaire des particularismes nationaux (Algérie, Égypte, Jordanie, Yémen, éléments pour l'analyse) n'est traitée ici que sous forme d'annonce de plan.

\section{NOTES}

1. Pour un repérage des positions de la doctrine de l'islam institutionnel contemporain à l'égard de la notion de pluralisme et de démocratie, cf. notamment la communication de Gudrun Kramer au colloque franco-égyptien de politologie (CEDEJ-CES) Le Caire, septembre 1990 (à paraître).

2. "Pour certains, la démocratie constitue un modale et une finalité. Historiquement, la démocratie n'est pas cela. Il faut revenir aux premiers philosophes grecs selon lesquels "démocratie "signifie amour du peuple. Pour ma part, j'estime que la démocratie est un moyen, pas une fin. Elle est un lieu de débat et non un modèle de réforme qui met fin à la crise économique, politique, culturelle et sociale (...). Ce régime se cache derrière ce concept car il n'ose pas avouer qu'il est communiste. Le communisme n'est pas dans ses plus beaux jours. Notre compréhension de la démocratie, c'est de permettre au peuple de donner son point de vue. Notre pays vit aujourd'hui une situation de crise politique. Le dialogue est donc pour nous le meilleur moyen d'aboutir. " Cf. également, sur ce registre, "Al-chûra, là al-dimocrâtiyya », Le Caire...

3. A tout le moins depuis 1981, date à laquelle son mouvement, alors Mouvement de la Tendance Islamique, s'est déclaré (pour la première/fois) officiellement favorable au pluralisme et s'est engagé à reconnaître toute majorité, «fût-elle communiste », qui résulterait d'un scrutin légal en Tunisie. Cf. le texte de la déclaration in F.B., L'Islamisme au Maghreb : la voix du Sud, op. cit.

4. Retranscription originale d'une interview effectuée pour Jeune Afrique Plus, juillet 1990.

5. Mais également chez une composante au moins du courant égyptien, le Parti $d u$ Travail d'Ibrahim Choukri notamment, au sein duquel les membres de la tendance islamiste ont pris la majorité depuis le Congrès de mars 1989 et qui défend une ligne comparable à celle du tunisien Ghannouchi. Cf. à ce propos les prises de position de M. 'Adil Husayn, rédacteur en chef de l'hebdomadaire AI-Chaab, organe du Parti du Travail, in «Nationalisme, communisme, islamisme : itinéraire d'un intellectuel égyptien ", Égypte/Monde Arabe ${ }^{\circ}{ }^{5}$, à paraître.

6. Cf. notamment Mohamed Arkoun in Maghreb Machreq, 1983 ou dans Ouverture sur l'Islam, Jacques Grancher Editeur, Paris, 1989.

7. Simple énoncé des expériences nationales qui mériteraient des analyses détaillées.

8. En Algérie en revanche, l'existence du principal référent anté- islamique (berbère) a été longuement occultée par le régime, amplifiant ainsi d'autant la propension de son « successeur » arabo-musulman à occuper tout l'espace de l'imaginaire social.

9. Cf. notamment Kilani Moussa, AI-Harakat al-islamiyya fi-l 'urdun, Dar al-bachir, Amman, 1989 et Mohamed Salim 'Ubaydat, Athar al-jamaât al-islamiyya al-maidânl khilal al-qarn al-achrîn, Maktabat al-rissalat al-hadîtha, Amman ; Mohsen Mohamed Salih, Al-tiar al-islâmî fi falastîn wa atharuhu fi harakat al-nahda, 1917-1948, Maktabat al-falâh lil nachr wal tawzi', Kuweit, 1989.

10. En septembre 1962, lorsque la dynastie des imams zaydites est détrônée par l'armée du Royaume, l'Imam déchu met sur pied une guérilla qui, soutenue par l'Arabie, Saoudite et la Grande-Bretagne, parvient six années durant à mettre en danger la jeune République. C'est dans 
ce contexte qu'est requis l'appui de l'armée de Nasser dont les soldats vont ainsi, pour beaucoup de ruraux du Nord, représenter, avec « les Turcs », le principal référent « étranger » de l'histoire contemporaine. Cf. Mohamed Yahia Al-Haddad, Tarikh al-Yemen al-siyassi, Manchourât al-Medina, Dar al-tanwir, Beyrouth, 1986 (4 ${ }^{\text {ème }}$ éd.) et François Burgat, "Le Yémen à l'heure de la réunification », Maghreb Machreq-Monde arabe (128), avril-mai- juin 1990, p.106.

INDEX

Mots-clés : démocratie, démocratisation, discours, islamisme, transition démocratique

\section{AUTEUR}

FRANÇOIS BURGAT

CEDEJ-CNRS 